PROCEEDINGS OF THE

AMERICAN MATHEMATICAL SOCIETY

Volume 131, Number 2, Pages 441-448

S 0002-9939(02)06783-7

Article electronically published on September 17, 2002

\title{
SOLITON SOLUTIONS FOR QUASILINEAR SCHRÖDINGER EQUATIONS, I
}

\author{
JIAQUAN LIU AND ZHI-QIANG WANG
}

(Communicated by David S. Tartakoff)

\begin{abstract}
For a class of quasilinear Schrödinger equations we establish the existence of ground states of soliton type solutions by a minimization argument.
\end{abstract}

\section{INTRODUCTION}

This paper is concerned with the existence of standing wave solutions for quasilinear Schrödinger equations of the form

$$
i \partial_{t} z=-\Delta z+W(x) z-f\left(|z|^{2}\right) z-\kappa \Delta h\left(|z|^{2}\right) h^{\prime}\left(|z|^{2}\right) z
$$

where $W(x), x \in \mathbf{R}^{N}$, is a given potential, $\kappa$ is a real constant and $f, h$ are real functions of essentially pure power forms. The semilinear case corresponding to $\kappa=0$ has been studied extensively in recent years (e.g., [3], [9], 24]). Quasilinear equations of form (11) appear more naturally in mathematical physics and have been derived as models of several physical phenomena corresponding to various types of $h$, the superfluid film equation in plasma physics by Kurihara in [13] (cf. [14]) for $h(s)=s$. In the case $h(s)=(1+s)^{1 / 2}$, (1) models the self-channeling of a high-power ultra short laser in matter; see [4, 6], 8], [23] and the references in 5]. Equation (1) also appears in plasma physics and fluid mechanics [13, [14, 17, [19], [21, in the theory of Heisenberg ferromagnets and magnons [2], [12], [15], 22., [25], in dissipative quantum mechanics [10] and in condensed matter theory [18]. In the mathematical literature very few results are known about equations of the form (1).

We consider the existence of standing wave solutions for quasilinear Schrödinger equations of form (1) with $h$ and $f$ as pure power functions of the dependent variable (though our method would apply to a more general type of nonlinearity). We consider the case $h(s)=s^{\alpha}, f(s)=\lambda s^{\frac{p-1}{2}}$ and $\kappa>0$. Putting $z(t)=\exp (-i F t) u(x)$ we obtain a corresponding equation of elliptic type which has a formal variational structure:

$$
-\Delta u+V(x) u-\alpha \kappa\left(\Delta\left(|u|^{2 \alpha}\right)\right)|u|^{2 \alpha-2} u=\lambda|u|^{p-1} u, \quad u>0, \quad \text { in } \mathbf{R}^{N},
$$

where $V(x)=W(x)-F$ is the new potential function. By a simple scaling, without loss of generality, we may assume $\alpha \kappa=1$ (corresponding to a new $\lambda$ ) throughout the paper. In the following we always assume $V \in C\left(\mathbf{R}^{N}, \mathbf{R}\right)$ and $\inf _{\mathbf{R}^{N}} V(x)>0$. We

Received by the editors September 4, 2001.

2000 Mathematics Subject Classification. Primary 35J10, 35J20, 35J25. 
also assume $N \geq 2$ since results for $N=1$ have been given in [20]. Let $2^{*}=\frac{2 N}{N-2}$ for $N \geq 3$ and $2^{*}=\infty$ for $N=2$. We consider several types of potentials.

(V1) $\lim _{|x| \rightarrow \infty} V(x)=+\infty$.

(V2) $V$ is radially symmetric, i.e., $V(x)=V(|x|)$.

(V3) $V$ is periodic in each variable of $x_{1}, \cdots, x_{N}$.

(V4) $V_{\infty}:=\lim _{|x| \rightarrow \infty} V(x)=\|V\|_{L^{\infty}\left(\mathbf{R}^{N}\right)}<\infty$.

Theorem 1.1 (The compact case). Let $\alpha>\frac{1}{2}$ and $2<p+1<2 \alpha 2^{*}$. Assume (V1) or (V2). Then there exist $\lambda_{n} \rightarrow \infty$ such that (2) has a solution. If in addition $4 \alpha \leq p+1$, there also exist $\lambda_{n} \rightarrow 0$ such that (2) has a solution.

Theorem 1.2 (The locally compact case). Let $\alpha>\frac{1}{2}$ and $4 \alpha \leq p+1<2 \alpha 2^{*}$. Assume (V3) or (V4). Then (2) has a solution for a sequence of $\lambda_{n} \rightarrow \infty$ and a sequence of $\lambda_{n} \rightarrow 0$.

Remark 1.3. The second author has given results on (2) in [20. We emphasize here that for $\alpha>\frac{1}{2}, 2 \alpha 2^{*}>2^{*}$ and the condition $4 \alpha \leq p+1<2 \alpha 2^{*}$ can be satisfied for all dimensions $N$. The result in Theorem 1.1 was proved in [20] for the case: $N=1, \alpha=1,2<p+1$; and the result in Theorem 1.2 was proved in 20 under the assumptions: $N=1, \alpha=1,4 \leq p+1$.

Remark 1.4. It would be interesting to know whether solutions exist for all $\lambda>0$ in (2). We shall discuss this in a forthcoming paper.

Theorems 1.1 and 1.2 will be proved in Sections 2 and 3, respectively.

\section{Ground state solutions - The Compact Case}

We consider a family of minimization problems, for $a>0$

$$
m_{a}=\inf _{M_{a}} E(u)
$$

where

$$
\begin{gathered}
M_{a}=\left\{u \in X \mid\|u\|_{p+1}=a\right\} \\
E(u)=\int_{\mathbf{R}^{N}}\left(|\nabla u|^{2}+V u^{2}\right) d x+2 \alpha \int_{\mathbf{R}^{N}}|u|^{2(2 \alpha-1)}|\nabla u|^{2} d x
\end{gathered}
$$

and in case (V1)

$$
X=\left\{u \in H^{1,2}\left(\mathbf{R}^{N}\right) \mid \int_{\mathbf{R}^{N}} V(x) u^{2} d x<\infty\right\}
$$

with norm given by $\|u\|^{2}=\int_{\mathbf{R}^{N}}\left(|\nabla u|^{2}+V u^{2}\right) d x$, and in case (V2)

$$
X=H_{r}^{1,2}\left(\mathbf{R}^{N}\right)=\left\{u \in H^{1,2}\left(\mathbf{R}^{N}\right) \mid u \text { is radial }\right\} .
$$

In both cases, $X$ is a subspace of $H^{1,2}\left(\mathbf{R}^{N}\right)$. We also need another Sobolev space $D^{1,2}\left(\mathbf{R}^{N}\right)$ for $N \geq 3$ which is the completion of $C_{0}^{\infty}\left(\mathbf{R}^{N}\right)$ under the norm $\|u\|^{2}=\int_{\mathbf{R}^{N}}|\nabla u|^{2} d x$. By the Sobolev inequality, $D^{1,2}\left(\mathbf{R}^{N}\right)$ is continuously embedded into $L^{2^{*}}\left(\mathbf{R}^{N}\right)$. Solutions of (2) will be shown to exist as minimizers of the above minimization problems which are called ground state solutions of (2)). Under (V1) or (V2) we have that the embedding from $X$ into $L^{p+1}\left(\mathbf{R}^{N}\right)$ is compact (e.g., [1], [11], [24], [26]). 
Lemma 2.1. For all $a>0, m_{a}$ is achieved at some $u_{a} \in M_{a}$ which is a weak solution of equation (2) with $\lambda=\lambda_{a}$ satisfying $\lambda_{a} \in\left(\frac{m_{a}}{a^{p+1}}, \frac{2 \alpha m_{a}}{a^{p+1}}\right)$.

Proof. We fix $a>0$. Let $\left(u_{n}\right) \in M_{a}$ be a minimizing sequence for $m_{a}$. Then by the compact embedding result from $X$ into $L^{q}\left(\mathbf{R}^{N}\right)$ for $2 \leq q<2^{*}$, we first have $u_{n} \rightarrow u$ in $X$ (weak convergence) and $u_{n} \rightarrow u$ in $L^{q}\left(\mathbf{R}^{N}\right)$ for $2 \leq q<2^{*}$. Since $\nabla\left(u_{n}^{2 \alpha}\right)$ is uniformly bounded in $L^{2}\left(\mathbf{R}^{N}\right)$, by the Sobolev inequality we have $\left\|u_{n}^{2 \alpha}\right\|_{2^{*}} \leq C$, which gives $\left\|u_{n}\right\|_{2 \alpha 2^{*}} \leq C$. By Hölder inequality we have $u_{n} \rightarrow u$ in $L^{q}\left(\mathbf{R}^{N}\right)$ for $2 \leq q<2 \alpha 2^{*}$. Then we claim

$$
\varliminf_{n \rightarrow \infty} \int_{\mathbf{R}^{N}}\left(\left|\nabla u_{n}\right|^{2}+V u_{n}^{2}\right) d x+2 \alpha \int_{\mathbf{R}^{N}}\left|u_{n}\right|^{2(2 \alpha-1)}\left|\nabla u_{n}\right|^{2} d x \geq E(u) .
$$

To see this let us observe that the first integral is the norm of $u_{n}$ in $X$ which makes it weakly lower semi-continuous in $X$. The second integral in $E\left(u_{n}\right)$ can be regarded as the $D^{1,2}\left(\mathbf{R}^{N}\right)$ norm of $v_{n}=\left(u_{n}\right)^{2 \alpha}$ when $N \geq 3$. Since $v_{n}$ is bounded in $D^{1,2}\left(\mathbf{R}^{N}\right)$ there is $v \in D^{1,2}\left(\mathbf{R}^{N}\right)$ such that $v_{n} \rightarrow v$ in $D^{1,2}\left(\mathbf{R}^{N}\right)$ (weak convergence). Also we may assert that $u_{n} \rightarrow u$ a.e. in $\mathbf{R}^{N}$ and $v_{n} \rightarrow v$ a.e. in $\mathbf{R}^{N}$. From this we have $v=u^{2 \alpha}$. Therefore the second integral is bounded from below by $\int_{\mathbf{R}^{N}}|u|^{2(2 \alpha-1)}|\nabla u|^{2} d x$. Thus we have proved the claim when $N \geq 3$. For $N=2$, we use the continuous embedding $X$ into $L^{q}\left(\mathbf{R}^{N}\right)$ for any $2 \leq q<\infty$ (instead of using $D^{1,2}\left(\mathbf{R}^{N}\right)$ ) and a similar argument works. Hence we obtain that $m_{a}$ is achieved at some $u \in M_{a}$. Since we may assume $u_{n} \geq 0$ we have $u \geq 0$. By the Lagrange multiplier theorem ([7]) we conclude that $u$ is a weak solution of (2)

$$
-\Delta u+V(x) u-\left(\Delta\left(|u|^{2 \alpha}\right)\right)|u|^{2 \alpha-2} u=\lambda_{a}|u|^{p-1} u, \text { in } \mathbf{R}^{N},
$$

where $\lambda_{a}$ is the Lagrange multiplier. Multiplying the equation by $u$ and integrating over $\mathbf{R}^{N}$ we get

$$
\lambda_{a} \in\left(\frac{m_{a}}{a^{p+1}}, \frac{2 \alpha m_{a}}{a^{p+1}}\right) .
$$

In order to show there exist $\lambda_{n} \rightarrow \infty$ and $\lambda_{n} \rightarrow 0$ such that (2) has a solution, we need the following lemma which does not depend on (V1) and (V2) and will also be used again later.

Lemma 2.2. Assume $m_{a}$ is achieved for all $a>0$. Let $u_{a}$ be a minimizer and $\lambda_{a}$ the corresponding Lagrange multiplier. Then $\lambda_{a} \rightarrow \infty$ as $a \rightarrow 0$ when $2<p+1$, and $\lambda_{a} \rightarrow 0$ as $a \rightarrow \infty$ when $4 \alpha \leq p+1$.

Proof. To show $\lambda_{a} \rightarrow \infty$ as $a \rightarrow 0$ we assume to the contrary that there exist $a_{n} \rightarrow 0$ such that $\lambda_{n}=\lambda_{a_{n}} \leq C_{1}$. By (2) we have $u_{n}$ uniformly bounded in $X$ and $v_{n}=u_{n}^{2 \alpha}$ uniformly bounded in $D^{1,2}\left(\mathbf{R}^{N}\right)$, so by embedding theorems we have $u_{n}$ bounded in $L^{q}\left(\mathbf{R}^{N}\right)$ for any $2 \leq q \leq 2 \alpha 2^{*}$. Especially, $u_{n}$ is bounded in $L^{p+1}\left(\mathbf{R}^{N}\right)$ and we have with $s$ satisfying $\frac{1}{p+1}=\frac{s}{2}+\frac{1-s}{2 \alpha 2^{*}}$

$$
\begin{aligned}
& \left\|u_{n}\right\|_{p+1}^{p+1} \\
\leq & \left\|u_{n}\right\|_{2}^{s(p+1)}\left\|u_{n}\right\|_{2 \alpha 2^{*}}^{(1-s)(p+1)} \\
\leq & C\left(\left\|u_{n}\right\|_{X}^{p+1}+\left\|u_{n}^{2 \alpha}\right\|_{2^{*}}^{2 \alpha(p+1)}\right) \\
\leq & C\left(\left\|u_{n}\right\|_{X}^{p+1}+\left\|\nabla\left(u_{n}^{2 \alpha}\right)\right\|_{2}^{2 \alpha(p+1)}\right) .
\end{aligned}
$$


Here $C$ is a constant independent of $n$. On the other hand, using $\left\|u_{n}\right\|_{p+1}=a_{n} \rightarrow 0$ and the equation, we have

$$
\left\|u_{n}\right\|_{X}^{2}+(2 \alpha)^{2} \int_{\mathbf{R}^{N}}\left|\nabla u_{n}^{2 \alpha}\right|^{2} d x=\lambda_{n} \int_{\mathbf{R}^{N}}\left|u_{n}\right|^{p+1} d x \leq C_{1} a_{n}^{p+1} \rightarrow 0 .
$$

Then using $\alpha(p+1)>\frac{p+1}{2}$ and the above two inequalities we have

$$
\begin{aligned}
& \left\|u_{n}\right\|_{X}^{2}+(2 \alpha)^{2} \int_{\mathbf{R}^{N}}\left|\nabla u_{n}^{2 \alpha}\right|^{2} d x \\
= & \lambda_{n} \int_{\mathbf{R}^{N}}\left|u_{n}\right|^{p+1} d x \\
\leq & C_{1} C\left(\left\|u_{n}\right\|_{X}^{2}+\int_{\mathbf{R}^{N}}\left|\nabla u_{n}^{2 \alpha}\right|^{2} d x\right)^{\frac{p+1}{2}}
\end{aligned}
$$

where $C$ is independent of $n$. This implies $\left\|u_{n}\right\|_{X}^{2}+\int_{\mathbf{R}^{N}}\left|\nabla u_{n}^{2 \alpha}\right|^{2} d x \geq C_{2}$ for some $C_{2}>0$, for $p+1>2$. This is a contradiction with $\left\|u_{n}\right\|_{X}^{2}+\int_{\mathbf{R}^{N}}\left|\nabla u_{n}^{2 \alpha}\right|^{2} d x \rightarrow 0$.

Next, we show that if $p+1 \geq 4 \alpha, \lambda_{a} \rightarrow 0$ as $a \rightarrow \infty$. To see this, let us observe that if $u \in M_{1}, a u \in M_{a}$. Then by a simple scaling argument, for $a \geq 1$ we have

$$
m_{a} \leq a^{4 \alpha} m_{1}
$$

and for $a \leq 1$

$$
m_{a} \geq a^{4 \alpha} m_{1}
$$

Then using (6), for $4 \alpha<p+1$ we have as $a \rightarrow \infty$

$$
\lambda_{a} \leq \frac{2 \alpha m_{a}}{a^{p+1}} \leq 2 \alpha m_{1} a^{4 \alpha-(p+1)} \rightarrow 0 .
$$

The case of $p+1=4 \alpha$ is treated next. For any $\epsilon>0$, we first choose $u_{\epsilon} \in C_{0}^{\infty}\left(\mathbf{R}^{N}\right)$ such that $2 \alpha \int_{\mathbf{R}^{N}}\left|u_{\epsilon}\right|^{2(2 \alpha-1)}\left|\nabla u_{\epsilon}\right|^{2} d x<\epsilon \int_{\mathbf{R}^{N}}\left|u_{\epsilon}\right|^{p+1} d x$. This can be obtained by choosing $u_{\epsilon}(x)=u_{0}(\epsilon x)$ for some $u_{0} \in C_{0}^{\infty}\left(\mathbf{R}^{N}\right)$ with $\epsilon$ small. We may assume $\left\|u_{\epsilon}\right\|_{p+1}=1$ (since $p+1=4 \alpha$ ) so that $a u_{\epsilon} \in M_{a}$. Then since $p+1=4 \alpha>2$, for $a$ large we have

(9)

$$
\begin{aligned}
& m_{a} \leq E\left(a u_{\epsilon}\right) \\
& \leq a^{2} \int_{\mathbf{R}^{N}}\left(\left|\nabla u_{\epsilon}\right|^{2}+V u_{\epsilon}^{2}\right) d x+2 \alpha a^{4 \alpha} \int_{\mathbf{R}^{N}}\left|u_{\epsilon}\right|^{2(2 \alpha-1)}\left|\nabla u_{\epsilon}\right|^{2} d x-\epsilon a^{4 \alpha}+\epsilon a^{4 \alpha} \\
& \leq \epsilon a^{4 \alpha} .
\end{aligned}
$$

This shows $\lim _{a \rightarrow \infty} \frac{m_{a}}{a^{4 \alpha}}=0$. Then using (6) we get $\lambda_{a} \rightarrow 0$ as $a \rightarrow \infty$.

Proof of Theorem 1.1. Theorem 1.1 follows from the last two lemmas.

Remark 2.3. (V1) can be replaced by any condition that guarantees the compact embedding from $X$ into $L^{p+1}\left(\mathbf{R}^{N}\right)$; see for example [1], [1].

\section{Ground state solutions - The locally compact case}

In this section, we consider the cases where the potential $V$ satisfies (V3) or (V4). The space $X$ is taken as $H^{1,2}\left(\mathbf{R}^{N}\right) . \quad M_{a}$ and $m_{a}$ can be defined as in the last section. Due to Lemma 2.2 we only need to prove that $m_{a}$ is achieved at some $u_{a} \in M_{a}$. Since the proof is the same we just treat the case $a=1$ and we write $m_{1}=m$ and $M_{1}=M$ for simplicity. 
Lemma 3.1. Let $\left(u_{n}\right) \subset M$ be a minimizing sequence for $m$. Then there is $\beta \in$ $(0,1]$ and $x_{n} \in \mathbf{R}^{N}$ such that for any $\epsilon>0$ there exists $R>0$, for any $R^{\prime} \geq R$

$$
\varliminf_{n \rightarrow \infty} \int_{B_{R}\left(x_{n}\right)}\left|u_{n}\right|^{p+1} d x \geq \beta-\epsilon
$$

and

$$
\varliminf_{n \rightarrow \infty} \int_{\mathbf{R}^{N} \backslash B_{R^{\prime}}\left(x_{n}\right)}\left|u_{n}\right|^{p+1} d x \geq(1-\beta)-\epsilon .
$$

Proof. For a minimizing sequence $u_{n}$ we have that $\int_{\mathbf{R}^{N}}\left|u_{n}\right|^{2(2 \alpha-1)}\left|\nabla u_{n}\right|^{2}$ is bounded which implies that $\int_{\mathbf{R}^{N}}\left|\nabla u_{n}^{2 \alpha}\right|^{2}$ is bounded. Thus $u_{n}^{2 \alpha}$ is uniformly bounded in $D^{1,2}\left(\mathbf{R}^{N}\right)$, and we have that $u_{n}$ is uniformly bounded in $L^{2 \alpha 2^{*}}\left(\mathbf{R}^{N}\right)$ by the Sobolev embedding. Also because $u_{n}$ is bounded in $L^{2}\left(\mathbf{R}^{N}\right)$ we get that $u_{n}$ is bounded in $L^{q}\left(\mathbf{R}^{N}\right)$ for all $q \in\left[2,2 \alpha 2^{*}\right]$ by the Hölder inequality. Especially, $v_{n}=u_{n}^{2 \alpha}$ is bounded in $L^{2}\left(\mathbf{R}^{N}\right)$. This implies that $u_{n}^{2 \alpha}$ is bounded in $H^{1}\left(\mathbf{R}^{N}\right)$. Note that $\left\|v_{n}\right\|_{\frac{p+1}{2 \alpha}}=1$. By P.L. Lions' Lemma $([16])$, there is $\beta \in(0,1]$ and $x_{n} \in \mathbf{R}^{N}$ such that for any $\epsilon>0$ there exists $R>0$ such that for any $R^{\prime} \geq R$, as $n \rightarrow \infty$,

$$
\int_{B_{R}\left(x_{n}\right)}\left|u_{n}\right|^{p+1} d x=\int_{B_{R}\left(x_{n}\right)}\left|v_{n}\right|^{\frac{p+1}{2 \alpha}} d x \geq \beta-\epsilon
$$

and

$$
\int_{\mathbf{R}^{N} \backslash B_{R^{\prime}}\left(x_{n}\right)}\left|u_{n}\right|^{p+1} d x=\int_{\mathbf{R}^{N} \backslash B_{R^{\prime}}\left(x_{n}\right)}\left|v_{n}\right|^{\frac{p+1}{2 \alpha}} \geq(1-\beta)-\epsilon .
$$

Proof of Theorem 1.2. We first consider the case (V3). Let $u_{n}$ be a minimizing sequence. From Lemma 3.1, we get $\beta \in(0,1]$, and a sequence $x_{n}$ such that (10) and (11) hold. We may assume the components of $x_{n}$ are integer multiples of the periods of $V(x)$. Thus $u_{n}\left(\cdot+x_{n}\right)$ is still a minimizing sequence. If $\beta=1$ we get a strong convergence of $u_{n}\left(\cdot+x_{n}\right) \rightarrow u$ in $L^{p+1}\left(\mathbf{R}^{N}\right)$, and a similar argument to that in the proof of Theorem 1.1 finishes the proof. If $\beta<1$ we derive a contradiction as follows. For $\epsilon>0$ and $R>0$ given in Lemma 3.1 let $\eta_{R}(t)$ be a smooth function defined on $[0, \infty)$ satisfying $\eta_{R}(t)=1$ for $t \leq R$ and $\eta_{R}(t)=0$ for $t \geq 2 R$ and $\eta_{R}^{\prime}(t) \leq \frac{2}{R}$. Let $\eta_{R}^{c}(t)=1-\eta_{R}(t)$. Define

$$
v_{n}(x)=\eta_{R}\left(\left|x-x_{n}\right|\right) u_{n}(x) \text { and } w_{n}(x)=\eta_{R}^{c}\left(\left|x-x_{n}\right|\right) u_{n}(x) .
$$

Then it is easy to see for $n$ large

$$
\left.\left|\int\right| v_{n}\right|^{p+1} d x-\beta \mid \leq \epsilon
$$

and

$$
\left.\left|\int\right| w_{n}\right|^{p+1} d x-(1-\beta) \mid \leq \epsilon
$$


Also a direct computation shows that

$$
\begin{aligned}
& \int_{\mathbf{R}^{N}}\left(\left|\nabla u_{n}\right|^{2}+V u_{n}^{2}\right) d x+2 \alpha \int_{\mathbf{R}^{N}}\left|u_{n}\right|^{2(2 \alpha-1)}\left|\nabla u_{n}\right|^{2} d x \\
& \geq \int_{\mathbf{R}^{N}}\left(\left|\nabla v_{n}\right|^{2}+V v_{n}^{2}\right) d x+2 \alpha \int_{\mathbf{R}^{N}}\left|v_{n}\right|^{2(2 \alpha-1)}\left|\nabla v_{n}\right|^{2} d x \\
& \quad+\int_{\mathbf{R}^{N}}\left(\left|\nabla w_{n}\right|^{2}+V w_{n}^{2}\right) d x+2 \alpha \int_{\mathbf{R}^{N}}\left|w_{n}\right|^{2(2 \alpha-1)}\left|\nabla w_{n}\right|^{2} d x-\frac{C}{R},
\end{aligned}
$$

where $C>0$ is independent of $n, \epsilon, R$. Assume $u_{n}\left(\cdot+x_{n}\right)$ converges weakly to $u$ in $X$ and let $u_{R}=\left.u\right|_{B_{R}(0)}$. Then $u_{R} \neq 0$ for $R$ large because $\beta>0$. Then by the fact $p+1 \geq 4 \alpha>2$, we have

$$
\begin{aligned}
& m+o(1) \\
= & E\left(u_{n}\right) \\
\geq & \left\|v_{n}\right\|_{p+1}^{4 \alpha} E\left(v_{n} /\left\|v_{n}\right\|_{p+1}\right)+\left\|w_{n}\right\|_{p+1}^{4 \alpha} E\left(w_{n} /\left\|w_{n}\right\|_{p+1}\right) \\
& +\left(\left\|v_{n}\right\|_{p+1}^{2}-\left\|v_{n}\right\|_{p+1}^{4 \alpha}\right) \int_{\mathbf{R}^{N}}\left(\left|\nabla v_{n}\right|^{2}+V v_{n}^{2}\right) \\
& +\left(\left\|w_{n}\right\|_{p+1}^{2}-\left\|w_{n}\right\|_{p+1}^{4 \alpha}\right) \int_{\mathbf{R}^{N}}\left(\left|\nabla w_{n}\right|^{2}+V w_{n}^{2}\right)-\frac{C}{R} \\
\geq & m\left(\left\|v_{n}\right\|_{p+1}^{4 \alpha}+\left\|w_{n}\right\|_{p+1}^{4 \alpha}\right)+\left(\left\|u_{R}\right\|_{p+1}^{2}-\left\|u_{R}\right\|_{p+1}^{4 \alpha}\right) \int_{\mathbf{R}^{N}}\left(\left|\nabla u_{R}\right|^{2}+V u_{R}^{2}\right)-\frac{C}{R} \\
\geq & m\left[(\beta-\epsilon)^{\frac{4 \alpha}{p+1}}+(1-\beta-\epsilon)^{\frac{4 \alpha}{p+1}}\right] \\
& +\left(\left\|u_{R}\right\|_{p+1}^{2}-\left\|u_{R}\right\|_{p+1}^{4 \alpha}\right) \int_{\mathbf{R}^{N}}\left(\left|\nabla u_{R}\right|^{2}+V u_{R}^{2}\right)-\frac{C}{R} .
\end{aligned}
$$

Letting $n \rightarrow \infty$ and then $\epsilon \rightarrow 0$ (which implies $R \rightarrow \infty$ ), we get a contradiction for $p+1 \geq 4 \alpha$. This shows that $\beta=1$. This completes the proof for the case of (V3).

For the case of (V4) we again consider a minimizing sequence $u_{n} \subset M$. Applying Lemma 3.1 we get $\beta>0$ and $x_{n} \in \mathbf{R}^{N}$ such that for any $\epsilon>0$ there is $R>0$ such that (10) and (11) hold. We show here that $\beta=1$ and $x_{n}$ is bounded in $\mathbf{R}^{N}$, which together imply that $u_{n}$ converge strongly in $L^{p+1}\left(\mathbf{R}^{N}\right)$. We show $\beta=1$ first. Assume for contradiction $\beta<1$. Similar to the proofs above, we define $v_{n}$ and $w_{n}$ with $\epsilon$ and $R>0$. Let

$$
m_{\infty}=\inf _{u \in M}\left(\int_{\mathbf{R}^{N}}\left(|\nabla u|^{2}+V_{\infty} u^{2}\right) d x+2 \alpha \int_{\mathbf{R}^{N}}|u|^{2(2 \alpha-1)}|\nabla u|^{2} d x\right),
$$

i.e., the infimum of $E(u)$ over $M$ with $V(x)$ replaced by $V_{\infty}$ in $E(u)$. Then $m_{\infty}$ is achieved by the proof in the first half above since (V3) is satisfied by a constant, say at $u \in M$ which we may assume to be positive in $\mathbf{R}^{N} \backslash\{0\}$. Using this $u$ as a test function we can show that if $V(x)$ is not identically equal to $V_{\infty}$, then $m<m_{\infty}$. 
Now if $\beta \in(0,1)$, we can follow a similar argument as above to get a contradiction:

(14)

$$
\begin{aligned}
m & +o(1) \\
= & E\left(u_{n}\right) \\
\geq & \left\|v_{n}\right\|\left\|_{p+1}^{4 \alpha} E\left(v_{n} /\left\|v_{n}\right\|_{p+1}\right)+\right\| w_{n} \|_{p+1}^{4 \alpha} E\left(w_{n} /\left\|w_{n}\right\|_{p+1}\right) \\
& +\left(\left\|v_{n}\right\|_{p+1}^{2}-\left\|v_{n}\right\|_{p+1}^{4 \alpha}\right) \int_{\mathbf{R}^{N}}\left(\left|\nabla v_{n}\right|^{2}+V v_{n}^{2}\right) \\
& +\left(\left\|w_{n}\right\|_{p+1}^{2}-\left\|w_{n}\right\|_{p+1}^{4 \alpha}\right) \int_{\mathbf{R}^{N}}\left(\left|\nabla w_{n}\right|^{2}+V w_{n}^{2}\right)-\frac{C}{R} \\
\geq & m\left(\left\|v_{n}\right\|_{p+1}^{4 \alpha}+\left\|w_{n}\right\|_{p+1}^{4 \alpha}\right)+\left(\left\|u_{R}\right\|_{p+1}^{2}-\left\|u_{R}\right\|_{p+1}^{4 \alpha}\right) \int_{\mathbf{R}^{N}}\left(\left|\nabla u_{R}\right|^{2}+V u_{R}^{2}\right)-\frac{C}{R} \\
\geq & m\left[(\beta-\epsilon)^{\frac{4 \alpha}{p+1}}+(1-\beta-\epsilon)^{\frac{4 \alpha}{p+1}}\right] \\
& +\left(\left\|u_{R}\right\|_{p+1}^{2}-\left\|u_{R}\right\|_{p+1}^{4 \alpha}\right) \int_{\mathbf{R}^{N}}\left(\left|\nabla u_{R}\right|^{2}+V u_{R}^{2}\right)-\frac{C}{R}
\end{aligned}
$$

by sending $n \rightarrow \infty, \epsilon \rightarrow 0(R \rightarrow \infty)$. Thus, $\beta=1$.

Next, we assume that $\beta=1$ and $\left|x_{n}\right| \rightarrow \infty$ as $n \rightarrow \infty$. Then we have $w_{n} \rightarrow 0$ in $L^{2}\left(\mathbf{R}^{N}\right)$. Then as we send $n \rightarrow \infty, \epsilon \rightarrow 0$,

$$
\begin{aligned}
m+o(1) & =E\left(u_{n}\right) \\
& \geq\left\|v_{n}\right\|_{p+1}^{4 \alpha} E\left(v_{n} /\left\|v_{n}\right\|_{p+1}\right)-\frac{C}{R} \\
& \geq\left\|v_{n}\right\|_{p+1}^{4 \alpha} E_{\infty}\left(v_{n} /\left\|v_{n}\right\|_{p+1}\right)-\frac{C}{R} \\
& \geq m_{\infty},
\end{aligned}
$$

a contradiction with $m<m_{\infty}$. The proof is complete.

\section{REFERENCES}

[1] Bartsch, T., Wang, Z.-Q.: Existence and multiplicity results for some superlinear elliptic problems on $\mathbf{R}^{n}$. Comm. Partial Differential Equations 20, 1725-1741 (1995). MR 96f:35050

[2] Bass, F. G., Nasanov, N. N.: Nonlinear electromagnetic spin waves. Physics Reports 189, 165-223 (1990).

[3] Berestycki, H., Lions, P. L.: Nonlinear scalar field equations, I: Existence of a ground state. Arch. Rational Mech. Anal. 82, 313-346 (1983). MR 84h:35054a

[4] Borovskii, A. V., Galkin, A. L.: Dynamical modulation of an ultrashort high-intensity laser pulse in matter. JETP 77, 562-573 (1993).

[5] De Bouard, A., Hayashi, N., Saut, J.-C.: Global existence of small solutions to a relativistic nonlinear Schrödinger equation. Comm. Math. Phys. 189, 73-105 (1997). MR 98k:35174

[6] Brandi, H. S., Manus, C., Mainfray, G., Lehner, T., Bonnaud, G.: Relativistic and ponderomotive self-focusing of a laser beam in a radially inhomogeneous plasma. Phys. Fluids B 5, 3539-3550 (1993).

[7] Browder, F. E.: Variational methods for nonlinear elliptic eigenvalue problems. Bull. Amer. Math. Soc. 71, 176-183 (1965). MR 31:3707

[8] Chen, X. L., Sudan, R. N.: Necessary and sufficient conditions for self-focusing of short ultraintense laser pulse. Phys. Review Letters 70, 2082-2085 (1993)

[9] Floer, A., Weinstein, A.: Nonspreading wave packets for the cubic Schrödinger equation with a bounded potential. J. Funct. Anal. 69, 397-408 (1986). MR 88d:35169

[10] Hasse, R. W.: A general method for the solution of nonlinear soliton and kink Schrödinger equations. Z. Physik B 37, 83-87 (1980). MR 80m:81017 
[11] Kondrat'ev, V., Shubin, M.:, Discreteness of spectrum for the Schrödinger operator on manifolds of bounded geometry. Operator theory: Advances and Applications, 110 (1999), 185-226. MR 2001c:58030

[12] Kosevich, A. M., Ivanov, B. A., Kovalev, A. S.: Magnetic solitons. Physics Reports 194, 117-238 (1990)

[13] Kurihura, S.: Large-amplitude quasi-solitons in superfluid films. J. Phys. Soc. Japan 50, 3262-3267 (1981).

[14] Laedke, E. W., Spatschek, K. H., Stenflo, L.: Evolution theorem for a class of perturbed envelope soliton solutions. J. Math. Phys. 24, 2764-2769 (1983). MR 85e:76012

[15] Lange, H., Toomire, B., Zweifel, P. F.: Time-dependent dissipation in nonlinear Schrödinger systems. J. Math. Phys. 36, 1274-1283 (1995). MR 95k:35193

[16] Lions, P.-L.: Concentration compactness principle in the calculus of variations. The limit case. Part 1, Rev. Mat. Ibero., 1, 145-201 (1985). MR 87c:49007

[17] Litvak, A. G., Sergeev, A. M.: One dimensional collapse of plasma waves. JETP Letters 27, (1978) 517-520.

[18] Makhankov, V. G., Fedyanin, V. K.: Non-linear effects in quasi-one-dimensional models of condensed matter theory. Physics Reports 104, 1-86 (1984). MR 85b:82003

[19] Nakamura, A.: Damping and modification of exciton solitary waves. J. Phys. Soc. Japan 42, 1824-1835 (1977).

[20] Poppenberg, M., Schmitt, K., Wang, Z.-Q.: On the existence of soliton solutions to quasilinear Schrödinger equations. Calculus of Variations and PDEs 14, 329-344 (2002).

[21] Porkolab, M., Goldman, M. V.: Upper hybrid solitons and oscillating two-stream instabilities. Physics of Fluids 19, 872-881 (1976). MR 54:14658

[22] Quispel, G. R. W., Capel, H. W.: Equation of motion for the Heisenberg spin chain. Physica 110 A, 41-80 (1982). MR 83g:82071

[23] Ritchie, B.: Relativistic self-focusing and channel formation in laser-plasma interactions. Phys. Rev. E 50, 687-689 (1994).

[24] Strauss, W. A.:Existence of solitary waves in higher dimensions. Comm. Math. Phys., 55, 149-162 (1977). MR 56:12616

[25] Takeno, S., Homma, S.: Classical planar Heisenberg ferromagnet, complex scalar fields and nonlinear excitations. Progr. Theoret. Physics 65, 172-189 (1981).

[26] Willem, M.: Minimax Theorems. Birkhäuser, Boston, (1996). MR 97h:58037

Department of Mathematics, Peking University, Beijing, 100871, People's Republid OF CHINA

E-mail address: jiaquan@math.pku.edu.cn

Department of Mathematics and Statistics, Utah State University, Logan, Utah 84322

E-mail address: wang@math.usu.edu 